\title{
Ischemia Is the Prime but Not the Only Cause of Human Multipotent Stromal Cell Death in Tissue-Engineered Constructs In Vivo
}

To cite this article:

Pierre Becquart, Adeline Cambon-Binder, Laurent-Emmanuel Monfoulet, Marianne Bourguignon, Katleen Vandamme, Morad Bensidhoum, Hervé Petite, and Delphine Logeart-Avramoglou. Tissue Engineering Part A. October 2012, 18(19-20): 2084-2094. doi:10.1089/ten.tea.2011.0690.

Published in Volume: 18 Issue 19-20: October 1, 2012

Online Ahead of Print: June 25, 2012

Online Ahead of Editing: May 11, 2012

Author information

Pierre Becquart, M.S.,1,*

*These authors equally contributed to this work.

Adeline Cambon-Binder, M.D., M.S., ${ }^{1},{ }^{*}$ Laurent-Emmanuel Monfoulet, Ph.D., ${ }^{1}$ Marianne Bourguignon, M.S., ${ }^{1}$ Katleen Vandamme, Ph.D., ${ }^{2}$ Morad Bensidhoum, Ph.D., ${ }^{1}$ Hervé Petite, Ph.D., ${ }^{1}$ and Delphine Logeart-Avramoglou, Ph.D. ${ }^{1}$

${ }^{1}$ Laboratory of Bioengineering and Biomechanics for Bone and Articulations, UMR 7052 CNRS, Univ Paris Diderot, Sorbonne Paris Cité, Paris, France.

${ }^{2}$ Department of Prosthetic Dentistry, Biomat Research Cluster KU Leuven, Leuven, Belgium.

Address correspondence to:

Delphine Logeart-Avramoglou, Ph.D.

Laboratory of Bioengineering and Biomechanics for Bone and Articulations

UMR 7052 CNRS

Univ Paris Diderot

Sorbonne Paris Cité

10 Ave., de Verdun

75010 Paris

France

E-mail: delphine.logeart@univ-paris-diderot.fr

Received: December 7, 2011

Accepted: May 2, 2012 


\section{ABSTRACT}

Local tissue ischemia is a prime cause responsible for the massive cell death in tissue-engineered (TE) constructs observed postimplantation. To assess the impact of ischemia on the death of implanted human multipotent stromal cells (hMSCs), which have great potential for repairing damaged tissues, we hereby investigated the in vivo temporal and spatial fate of human Luc-GFP-labeled MSCs within fibrin gel/coral scaffolds subcutaneously implanted in nude mice. In vivo bioluminescence imaging monitoring and histological analyses of the constructs tested confirmed the irremediable death of hMSCs over 30 days postimplantation. The kinetics of expression of three hypoxic/ischemic markers (HIF-1 $\alpha$, LDH-A, and BNIP3) was also monitored. Our results provided evidence that hMSCs located within the core of implanted constructs died faster and predominantly and strongly expressed the aforementioned ischemic markers. In contrast, cells located in the outer regions of TE constructs were reperfused by neovascularization and were still viable (as evidenced by their ex-vivo proliferative potential) at day 15 postimplantation. These results support the explanation that in the central part of the constructs tested, death of hMSCs was due to ischemia, whereas in the periphery of these constructs, cell death was due to another mechanism that needs to be elucidated. 


\section{Introduction}

Tissue engineering aims at regenerating functional tissues by combining cells with a supporting substrate. Owing to its expansion potential, ability to adopt different phenotypes, paracrine effects, and immune-modulatory properties, multipotent stromal cell (MSC) 1 represents a promising cell population for repairing damaged tissues such as bone,2,3 cartilage,4 kidney, muscle, skin, neural, and cardiac tissues.5,6 However, the efficacy of MSC-based TE in experimental and clinical studies has been less than optimal. For example, when addressing the repair of massive bone defects in a clinically relevant segmental bone defect model, the osteogenic ability of tissue constructs was superior to the one of scaffold alone but remained inferior to that of autologous bone grafts, the benchmark for bone repair.7

The underlying reasons for the limited efficacy of tissue constructs are not yet fully understood but one of them is the massive disappearance of transplanted cells during the first weeks postimplantation. Indeed, using different cellular and molecular labeling approaches to track implanted cells, several researchers reported the loss (from $70 \%$ to $100 \%$ ) of either animal- or human-derived MSCs (hMSCs) loaded into scaffolds and implanted either subcutaneously8-10 or into skeletal bone defects11-13 in rodent models within the first month after implantation. Although the mechanisms or biological cascade behind such extensive loss of implanted cells remains poorly understood, the hostile environment that MSC faced when loaded on synthetic material constructs devoid of pre-existing vascular network14 is considered as a prime reason. Upon implantation, MSCs encounter an ischemic environment of low oxygen tension and nutrient deprivation that could lead to their massive death in situ.15 However, information regarding the cellular responses to the ischemic stress is crucially lacking in the tissue engineering field. For these reasons, the aim of the present study was to assess the impact of ischemia on the loss of hMSCs implanted in vivo. Because the intensity of the ischemic state that individual cells face varies with both their location throughout the construct (especially their distance from blood capillaries) and the postimplantation time, we hereby investigated the in vivo hMSCs fate at different levels of depth within TE constructs and at early, mid-, and late postimplantation time points.

A human MSC line16 was transduced using lentiviral vectors encoding both firefly luciferase and enhanced green fluorescent protein (eGFP) reporter genes under the control of a constitutively active promoter. Cell viability, after the labeled cells were seeded onto fibrin gel/coral ceramic scaffolds and subcutaneously implanted in nude mice, was monitored in each cell construct noninvasively by bioluminescence imaging (BLI) over a period of 30 days. Because an excessively large number of cells within the scaffolds may worsen the ischemic conditions at the implantation site, the effect of the number of transplanted cells in their survival was particularly addressed. To further assess the metabolic stress induced by the ischemic environment, immunohistochemistry analyses were performed to establish maps of either implanted cells, apoptotic cells, or hypoxiaresponsive cells via the expression of hypoxia-inducible factor- 1 alpha (HIF- $1 \alpha$ ) and of two downstream proteins: the lactate dehydrogenase-A (LDH-A) and Bcl-2/adenovirus E1B 19 kDainteracting protein 3 (BNIP3). LDH-A is a marker of an anaerobic environment since this enzyme converts pyruvate (derived from glucose) to lactate through anaerobic glycolysis.17 BNIP3 is a marker of cell nutrient stress 18 and is implicated in the induction of autophagy.19,20 In the present study, the progression of the expression of these markers was monitored within specific regional zones defined within cell constructs retrieved at early, mid-, and late postimplantation time points. 


\section{Materials and Methods}

Cell transduction

A human immortalized multipotent stromal line (hMSC line SV5616), which expresses characteristics of human MSCs, was used in the present study. The hMSC SV56 line expresses the stromal marker (48\% of Stro1 positive cells), and CD105 (SH2) and CD73 (SH3), two mesenchymal cell markers (>90\% positive cells). 16 The multipotential property of the hMSC line SV56 was established by the ability to differentiate toward osteogenic, adipogenic, and chondrogenic lineages (Supplementary Fig. S1; Supplementary Data are available online at www.liebertpub.com/tea).

HMSC line SV56 line was genetically modified using a bicistronic lentiviral vector encoding firefly luciferase (fLuc) and eGFP(pCHMWS-fLuc-T2A-eGFP). The T2A sequence ensured efficient coexpression of both reporter genes.21,22 The LV-fLuc-T2A-eGFP vectors were obtained from Dr. A. Ibrahimi (Katholieke Universiteit, Leuven, Belgium). The hMSCs were cultured in alpha-modified Eagle medium ( $\alpha$-MEM; Sigma) supplemented with $10 \%$ fetal calf serum $(F C S)$, penicillin $(100 \mathrm{U} / \mathrm{mL})$, and streptomycin sulfate $(100 \mu \mathrm{g} / \mathrm{mL}$; all chemicals from the PAA Laboratories) under standard cell culture conditions. The cells were transduced with lentiviruses (at the concentration of $0.64 \mathrm{pg}$ of p24 viral coat protein per cell) as previously described.23 The efficiency of transduction was assessed by quantifying fluorescent and bioluminescent signals and comparing them to those obtained from nontransfected cells. Flow cytometry analysis of GFP-positive cells provided evidence that $88 \%$ of the transduced cells (designed as Luc-GFP-hMSC) were transduced. Luciferase expression in Luc-GFPhMSC was linear with the cell number (ranging from 10 to 106 cells; $r=0.98$ ). Upon expansion, both Luc-GFP-hMSC and hMSC displayed similar proliferation rates (doubling times at $2.43 \pm 0.46$ and $2.36 \pm 0.04$ days for transduced and nontranduced hMSC, respectively). When cultured under expansion conditions over five passages (10 doubling populations), the Luc-GFP-hMSC luciferaserelated activity was constant.

\section{Preparation of cell-containing constructs}

Luc-GFP-hMSC were seeded on natural Porites coral (Biocoral ${ }^{\circledR}$ ) particles (600 to $1000 \mu \mathrm{m}$ diameter) donated by Inoteb, Inc. This material consisted of $99 \%$ calcium carbonate in the form of aragonite, had open, communicating pores with a mean diameter of $100-300 \mu \mathrm{m}$, and a porosity of $49 \% .24,25$ The proliferative potential of the hMSC SV56 cells seeded into coral scaffold was checked and was confirmed in vitro (Supplementary Fig. S2). Prior to cell seeding, aliquots of $40 \mathrm{mg}$ of Porites coral particles were sterilized by autoclaving. For the study of the in vivo monitoring of the survival of implanted cells by BLI, four different densities of Luc-GFP-hMSC (suspended in $100 \mu \mathrm{L} \alpha$-MEM containing $10 \% \mathrm{FCS}$, antibiotics, and $25 \mathrm{mM} \mathrm{HEPES}$ ), were tested and delivered to each coral particle group: specifically, $5 \times 106$ ( $n=5$ constructs), $1 \times 106(n=6), 1 \times 105(n=6)$ and $1 \times 104(n=6)$ cells per cell construct. For the immunohistological studies of the constructs either nonimplanted (day 0 ) or explanted at day 6 , day 15 , and day 30 postimplantation, only three cell seeding densities were tested (specifically, $1 \times 106,1 \times 105$, and $1 \times 104$ cells per cell construct; $n=5$ constructs for each case). After two hours of incubation at $37^{\circ} \mathrm{C}$, the supernatant $(100 \mu \mathrm{L})$ was discarded and the particles were embedded in a fibrin gel (Tissucol ${ }^{\circledR}$; Baxter) as previously described.26 Briefly, first $100 \mu \mathrm{L}$ of fibrinogen $(18 \mathrm{mg} / \mathrm{mL})$, and then $5 \mu \mathrm{L}$ of thrombin $(100 \mathrm{IU} / \mathrm{mL})$ were added to the cell-seeded scaffold particles. The constructs used in the present study had a diameter of $0.5 \mathrm{~cm}$. These cell constructs were then maintained in $2 \mathrm{~mL}$ of standard growth medium at $37^{\circ} \mathrm{C}$ overnight. Cells contained in the supernatant of each construct were counted using a Coulter counter (BeckmanCoultronics). The results confirmed that the cells were properly loaded on the constructs tested (data not shown). Unseeded scaffolds served as negative controls. To control the efficacy of cell seeding, 
each construct was imaged (as described in the "In vivo noninvasive BLI and image analysis" section) in vitro just before implantation in animals. Analysis of the BLI images of Luc-GFP-hMSC-containing constructs provided evidence of a linear relationship ( $r 2=0.98)$ between the level of detected photons and the number of cells present in each construct (Supplementary Fig. S3).

In vivo implantation

Ten-week-old female nude mice were obtained from Janvier and handled according to the "European Guidelines for Care and Use of Laboratory Animals" (EEC Directives 86/609/CEE of 24.11.1986). All experimental animal procedures conducted in the present study were approved by the Ethics Committee on Animal Research of Lariboisiere/Villemin, Paris, France. One day after preparation of the cell-containing constructs (as described in the "Preparation of cell-containing constructs" section), each mouse was preoperatively given analgesics via subcuteneous injection of buprenorphine $(0.4 \mathrm{mg} / \mathrm{kg}$; Axience, Pautin, France) and was then anesthetized by intra-peritoneal injection of $100 \mathrm{mg} / \mathrm{kg}$ ketamine (Ketalar ${ }^{\circledR}$; Panpharma, Virbach) and $10 \mathrm{mg} / \mathrm{kg}$ xylazine (Rompun ${ }^{\circledR}$ $2 \%$; Bayer). The skin of the animals was prepared for surgery using povidone-iodine (Bétadine ${ }^{\circledR}$ ). Two symetrical incisions ( $0.5 \mathrm{~cm}$ in length) were made on the back of each mouse on both sides of the lumar spine and subcutaneous pouches were created. The cell-containing constructs of interest to the present study were carefully inserted in each pouch (two per mouse). The soft tissues at the implantation sites were then closed with two interrupted nonresorbable sutures. For the in vivo monitoring of implanted cell survival, the animals were then imaged (as described in the "In vivo noninvasive BLI and image analysis" section) twice a week before they were sacrificed using a pentobarbital overdose (Dolethal ${ }^{\circledR}$; Vetoquinol) at 30 days postimplantation. For the immunohistological studies, sets of animals were sacrificed at day 6 and 15 postimplantation. The constructs were explanted at that time and prepared for histological analysis, as described in the "Histology/immunohistology" section.

In vivo noninvasive $\mathrm{BLI}$ and image analysis

The mice tested in the present study were imaged twice a week during the 30-day postimplantation period as previously described.23 For this purpose, the mice were anesthetized by inhaling isoflurane. Thereafter, $50 \mu \mathrm{L}$ of D-luciferin $(20 \mathrm{mg} / \mathrm{mL}$ in phosphate-buffered saline [PBS]) were locally injected at each implantation site. After $10 \mathrm{~min}$ (and while still inhaling isoflurane), the animals were placed in the prone position inside the detection chamber of a BLI system (Ivis Lumina II ${ }^{\circledR}$; Caliper Life Sciences) and were imaged. Standard regions of interest surrounding each implant were manually delineated on the bioluminescence images and the photon flux emitted by each construct was quantified using the Living Image ${ }^{\circledR} 3.1$ software (Caliper Life Science).

\section{Histology/immunohistology}

At the end of the implantation time (specifically, 6, 15, and 30 days postimplantation), the implants were explanted and fixed in $4 \%$ paraformaldehyde $(\mathrm{pH} \mathrm{7.4)} \mathrm{for} 36 \mathrm{~h}$, decalcified in ethylenediaminetetraacetic acid $(14.5 \% \mathrm{w} / \mathrm{v})$ at $4^{\circ} \mathrm{C}$ for 1 week, and embedded in paraffin. A set of nonimplanted cell constructs were prepared in parallel (as described in the "Preparation of cellcontaining constructs"section) and were processed for histology (on day 0). Sequential sections (each $5 \mu \mathrm{m}$ thick) were either stained with hematoxylin-eosin-safranin (HES) or processed for immunodetection (for GFP, HIF-1 $\alpha$, LDHA, and BNIP3) or for terminal deoxyribonucleotidyl transferase-mediated dUTP nick end labeling (TUNEL). For immunodetection, tissue sections were paraffin dewaxed, rehydrated, and rinsed with $0.1 \mathrm{M} \mathrm{PBS}(\mathrm{pH}$ 7.4). These tissue sections were then pretreated either in a citrate buffer $(0.01 \mathrm{mM}, \mathrm{pH}=6)$ at $95^{\circ} \mathrm{C}$ for $20 \mathrm{~min}$, for subsequent HIF-1 $\alpha$, LDHA, and BNIP3 detection, or in a proteolytic enzyme solution (Dako) at $37^{\circ} \mathrm{C}$ for $20 \mathrm{~min}$ for 
subsequent GFP detection. Cell membranes were permeabilized using $0.3 \%$ Triton X-100 for 15 min; the endogenous peroxidase activity was inhibited by addition of peroxidase block (Kit Envision+; Dako) and the nonspecific binding sites were blocked using Protein Block (Dako). The tissue sections thus prepared were subsequently incubated in a humidified chamber in primary antibody (polyclonal rabbit anti-GFP 1/200 [ClonTech], polyclonal rabbit anti-HIF-1 $\alpha 1 / 100$ [Novus Biological], polyclonal rabbit anti-BNIP3 1/400 [Abcam], and in polyclonal rabbit anti-LDHA 1/1000, [Abcam]) for $1 \mathrm{~h}$, incubated with Labeled Polymer-HRP Anti-Rabbit antibody (Envision+ Kit; Dako K4011) for 30 min, and visualized using the DAB chromogen in the dark for 5 min (Envision+Kit; Dako K4011). Tissue sections from human epidermis biopsies, and tissue sections cell-containing constructs processed without the primary antibody were used as negative controls. Immunostaining for the HIF-1 $\alpha$ and BNIP3 proteins on human epidermis samples were undetectable while the LDH-A protein was only detected in the basal layer (Supplementary Fig. S4). Immunostaining for the HIF-1 $\alpha$, LDH-A, and BNIP3 proteins on tissue sections from unseeded constructs explanted at day 6 and day 15 postimplantation was also performed to assess expression of ischemic markers on cells coming from the invading host tissue and to determine any cross reaction of primary antibodies with scaffold components. The images obtained from these analyses showed the absence of cell labeling for the three proteins tested at both explantation time points. It should be noted that the anti-HIF-1 $\alpha$ staining on the construct explanted at day 6 exhibited strong nonspecific staining of the fibrin gel (Supplementary Fig. S5).

Detection of apoptotic cells, based on labeling of DNA strand breaks (TUNEL labeling), was performed using the "In situ cell-death detection kit" (Roche) and following the manufacturer's protocol. Briefly, tissue sections were paraffin dewaxed, rehydratated, permeabilized in a $0.1 \%$ sodium citrate $/ 0.1 \%$ Triton X-100 solution for $8 \mathrm{~min}$, and then incubated with labeling TUNEL (fluorescein-dUTP in $50 \mu \mathrm{L}$ per section) reaction mix, in the dark at $37^{\circ} \mathrm{C}$ for $1 \mathrm{~h}$. After rinsing with phosphate buffer thrice, the labeled specimens were maintained in $50 \mu \mathrm{L}$ of Converter-AP (anti-fluorescein-AP conjugate) in a humidified chamber at $37^{\circ} \mathrm{C}$ for $30 \mathrm{~min}$, rinsed twice with phosphate buffer, treated with $100 \mu \mathrm{L}$ $\mathrm{BCIP} / \mathrm{NBT}$ Substrate (Sigma Aldrich), and again maintained in the dark at $20^{\circ} \mathrm{C}$ for $10 \mathrm{~min}$. This labeling process was terminated by rinsing pertinent specimens with phosphate buffer twice. Specimens treated with $1 \mathrm{U} / \mathrm{mL}$ of DNAse (Invitrogen) were used as the positive control (Fig. 3; insert).

Histological analyses

To aid histological and immunohistochemical examination, regional analysis zones were defined within the implants, and the progression of events such as cell apoptosis and cell expression of hypoxic/ischemic markers were monitored within these zones. For TUNEL, anti-GFP, anti-HIF-1 $\alpha$, anti-LDHA, and anti-BNIP3 labeling, two stained sections from each scaffold (chosen at two different locations in the middle of each construct) were digitalized using a slide scanner (ScanScope ${ }^{\mathrm{TM}}$; Aperio). Images were collected using the TRIBVN ICS software (TRIBVN, Chatillon, France) and exported (*.tiff format) into photo imaging software (Coral Paint Shop Pro X) for analysis. For the GFP- and TUNEL-positive cells analyses, the periphery of each implant on the images of each tissue section of interest to the present study was delineated, then two other lines were drawn concentrically at a distance of $500 \mu \mathrm{m}$ and the other at $1000 \mu \mathrm{m}$ from, but along, the periphery of each section; in this fashion, outer, in-between, and inner areas were delineated, which represented $40 \%, 30 \%$, and $30 \%$ of the whole construct surface, respectively. The GFP- and TUNEL-positive cells in each of these areas were then counted. For the HIF-1 $\alpha$, LDHA, and BNIP3-positive cells analyses, three different fields located in the outer and inner area of each construct were examined (at 40xmagnification), and the number of labeled cells was counted and normalized over the total 
number of cells (both labeled and unlabeled corresponding to $>100$ cells) counted within the same field.

\section{Ex vivo analyses}

To further assess the viability of implanted cells, constructs seeded with $1 \times 106$ cells ( $n=8$ constructs) were explanted after 2, 6, and 15 days postimplantation. Cells (both surviving hMSCs and invading host cells) from each explant were isolated by enzymatic digestion (via incubation in a solution of collagenase type I $1 \%[\mathrm{w} / \mathrm{v}]$ and type IV $1 \%[\mathrm{w} / \mathrm{v}]$ in Hank's balanced salt solution medium at $37^{\circ} \mathrm{C}$ for $30 \mathrm{~min}$ ) of the constructs then seeded onto two wells of a six-well plate. Twenty-four hours after cell seeding, the cell cultures were washed twice with PBS (to eliminate remnant debris) and the cells were cultured in standard tissue culture medium. The growth kinetics of the explanted Luc-GFP hMSCs were monitored noninvasively by BLI after addition of $360 \mu \mathrm{g} / \mathrm{mL}$ luciferin substrate every 2 or 3 days after cell seeding till the cells reached confluence. To ensure that the presence of murine host cells do not impede the hMSC proliferation, and since the hMSC line SV56 cells contained the neomycin gene, 16 these cells were treated with $\mathrm{G} 418(400 \mu \mathrm{g} / \mathrm{mL})$.

\section{Statistical analyses}

Numerical results were reported as averagetstandard error of the mean. For in vivo experiments, multiple samples were analyzed for each group, specifically, $n=6$ constructs for the time course study and $n=5$ constructs for the histological analyses. Statistical analyses were conducted using the Statgraphics centurion version XV.2 (Statpoint, Inc.). Quantitative data from in vivo BLI signals were analyzed by two-way analysis of variance. The nonparametric Mann-Whitney $\mathrm{U}$ test was used to analyze data from two independent samples. For all analyses, differences at $p \leq 0.05$ were accepted to be statistically significant.

\section{Results}

Independent of cell seeding densities, implanted hMSCs died massively

The BLI signal from each implanted construct was monitored twice a week over the 30-day period of the study (Fig. 1A). To compare cell survival as a function of various cell seeding densities for each construct tested, photon fluxes were normalized with respect to the signal acquired at the beginning (day 1 -acquisition) of the in vivo period (Fig. 1B). The cell survival rate was significantly $(p<0.05)$ higher within the constructs seeded with $1 \times 106$ cells compared with the results obtained with the other cell densities tested till day 30 postimplantation. It should be noted that half of the implants seeded with $1 \times 106$ cells exhibited an increased BLI signal over the first 6 days postimplantation. No difference in cell survival rates was observed for the other cell densities tested during that time period. After day 6 , continuous decrease in the luminescence signal from all implanted cellcontaining constructs was observed; as a result, $<1 \%$ of the initial cell numbers for all constructs tested remained at 30 days postimplantation.

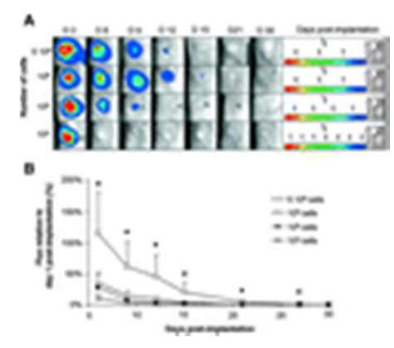


FIG. 1. In vivo bioluminescence imaging (BLI) monitoring of viability of cells in the constructs tested provided evidence of the massive death of implanted human multipotent stromal cell (hMSC)-Lucgreen fluorescent protein (GFP) at all cell seeding densities tested over 30 days of implantation. (A) Representative BLI images of cell-containing constructs subcutaneously implanted in nude mice. The rainbow pseudocolor scales were adjusted in all images for each cell seeding density group tested.

(B) Photon fluxes (normalized to those obtained at day 1 postimplantation for each construct tested) were plotted versus days postimplantation. $p<0.001$ (two-ways analysis of variance); ${ }^{*} p<0.05$ for the constructs seeded with $1 \times 106$ cells compared to constructs seeded with other cell densities (MannWhitney test). Color images available online at www.liebertpub.com/tea

This loss of hMSCs upon implantation was confirmed by the results obtained from GFP immunostaining of sections from cell-containing constructs either nonimplanted (day 0 ) or explanted at days 6,15 , and 30 postimplantation. Before implantation, the numbers of quantified GFP-cells were, however, underestimated because most of the seeded cells were closely adhered onto the scaffold surface and were not distributed within the fibrin gel as much as those observed postimplantation rendering the individual cell counting difficult. After implantation, the number of histologically visualized GFP-labeled cells was highest at day 6 postimplantation but decreased dramatically at day 15 and day 30 postimplantation. Only the constructs seeded with $1 \times 106$ cells still contained a significant $(p<0.01)$ number of GFP-labeled cells at day 15 postimplantation but few of the GFP-expressing cells remained at the end of the study period, that is 30 days postimplantation (Fig. 2A). Discrepancy was, however, noted between the results obtained from BLI detection and those from GFP cell counting at day 30 ( $<1 \%$ cell survival vs. $25 \%$ survival, respectively). Such a discrepancy could be due to the fact that BLI detection assesses the viability of implanted cells dynamically because of the short half-life $(\sim 2-4 h$ ) of the luciferin substrate; in contrast GFP (with half-life of several days) is a stable protein that may remain immunodetectable for few days after cell death.

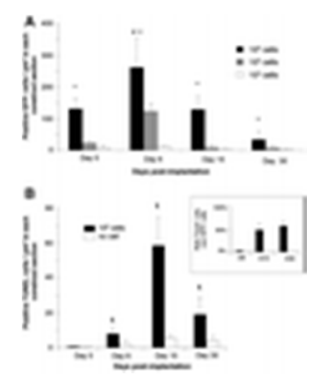

FIG. 2. Anti-GFP and TUNEL staining confirmed the death of implanted hMSCs. (A) Quantification of GFP-positive cells per $\mu \mathrm{m} 2$ detected per section from each cell construct either nonimplanted (day 0 ) or explanted at different time points postimplantation. (B) Time course of TUNEL-positive cells per $\mu \mathrm{m} 2$ detected per section from either unseeded constructs (white bars) or constructs seeded with $1 \times 106$ cells (black bars). Insert: Number of TUNEL-positive cells normalized to the number of GFPpositive cells detected on the same section from constructs seeded with $1 \times 106$ cells. Two stained sections from each construct were counted; $n=5$ constructs for each cell seeding density tested. The data were expressed as mean \pm standard error of the mean (SEM). ${ }^{*} p<0.05$ for the constructs seeded with $1 \times 106$ cells compared to constructs seeded with other cell densities (Mann-Whitney test); $\# p<0.05$ for the constructs analyzed at day 6 postimplantation compared to those analyzed at other time points (Mann-Whitney test); $\uparrow p<0.05$ for the constructs seeded with $1 \times 106$ cells compared to 
unseeded constructs (Mann-Whitney test). TUNEL, terminal deoxyribonucleotidyl transferasemediated dUTP nick end labeling.

To further understand the fate of hMSCs in the constructs seeded with $1 \times 106$ cells (the ones with the optimal in vivo viability), the number of apoptotic cells was also quantified on pertinent explants sections using TUNEL staining (Fig. 2B). Although no sign of cell apoptotis was observed at the time of implantation of the cell constructs, the number of TUNEL-positive cells increased at day 6 postimplantation, peaked at day 15 postimplantation (Fig. 2B), and was statistically different from the results obtained from the respective unseeded control group (which represented the average rate of apoptotic cells from the invading host tissue); at day 15 time point, the apoptotic cells represented $45 \%$ of the GFP-positive cells counted in the same construct section (Fig. 2B; insert). The number of TUNEL-positive cells decreased at day 30 postimplantation but still represented $57 \%$ of the GFP-positive cells.

Taken together, these data provided evidence that, although the seeding density of hMSCs affected cell survival at early times postimplantation, long-term viability of the implanted cells in TE constructs irremediably declined over time at all cell seeding densities tested; moreover, cell death by apoptosis peaked and remained elevated after day 15 postimplantation.

Implanted hMSCs died in the inner part of the construct first

To assess the impact of cell location within the TE constructs on the death of implanted cells, regions (specifically, outer, in-between, and inner areas) were defined within the explant sections, and the progression of events (such as disappearance of GFP-expressing cells and cell apoptosis) were monitored within each one of these zones.

GFP-labeled cells on individual sections from constructs seeded with $1 \times 106,1 \times 105$, and $1 \times 104$ cells were histologically visualized and quantified; the results from constructs seeded with $1 \times 106$ cells are representative of this analysis and are shown on Figure 3A, B, C, and D. Analysis of cell-containing but non implanted constructs (day 0 ) revealed that the seeded cells adhered onto the coral scaffold (Fig. $3 \mathrm{~A})$ and were uniformly distributed throughout the examined constructs as evidenced by the similar percentage of GFP-labeled cells present in each area (Fig. $3 \mathrm{H}$ ). At day 6 postimplantation, the GFPpositive cells had migrated within the fibrin gel (Fig. 3B) and were uniformly distributed throughout the sections for all cell seeding densities tested (Fig. 3H). At day 15 postimplantation, the remaining GFP-cells from constructs seeded with $1 \times 106$ and $1 \times 105$ cells were still evenly distributed throughout the respective constructs while cells from constructs seeded with $1 \times 104$ cells were located exclusively in the outer area $(p<0.05)$. After 30 days, there were no GFP-expressing cells of constructs seeded with $1 \times 105$ cells in both the inner and in-between areas $(p<0.05)$; in contrast, GFP-expressing cells of constructs seeded with $1 \times 106$ cells were still present in the core of the implant but displayed a necrotic/apoptotic aspect $(p<0.05)$.

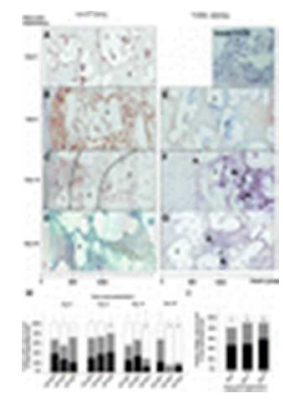


FIG. 3. Histology analysis revealed that implanted hMSCs in the inner part of the constructs died first. Sections from constructs seeded with $1 \times 106$ cells were labeled with anti-GFP immunostains (A$D)$ and with TUNEL labeling (E-G). These images show the implanted hMSCs and apoptotic cell distribution (black arrow heads) throughout the construct sections. The black lines separate the different areas of depth in the constructs: specifically, outer (from 0 to $500 \mu \mathrm{m}$ ), in-between (from 500 to $1000 \mu \mathrm{m}$ ), and inner (>1000 $\mu \mathrm{m}$ of depth). c, coral; f, fibrin. Insert: Sections treated with DNAse (positive control). (H) Percentage of GFP-positive cells present in the outer (white bars), inbetween (grey bars), and inner (black bars) areas delineated in each construct section. (I) Percentage of TUNEL-positive cells present in the outer (white bars), in-between (gray bars), and inner (black bars) areas in the delineated regions of the sections from constructs seeded with $1 \times 106$ cells. Two stained sections from each construct were examined; $n=5$ constructs for each cell seeding density tested. The data were expressed as mean \pm SEM. ${ }^{*} p<0.05$ for the positive cells present in the outer area compared to positive cells present in the in-between and inner areas (Mann-Whitney test). Color images available online at www.liebertpub.com/tea

Observation and analysis of TUNEL-stained sections from the constructs seeded with $1 \times 106$ cells confirmed that the apoptotic cells were mainly located in the core of the implant at all explantation time points tested (Fig. 3E-G). Specifically, $47 \%-59 \%$ and $30 \%-40 \%$ of the apoptotic cells were present in the inner and in-between areas, respectively (Fig. 3I). In contrast, a small number of apoptotic cells were present in the outer area at all postimplantation time points tested.

Altogether, these results provided evidence that implanted hMSCs died faster, and predominantly, when located in the inner part of the constructs; this effect was enhanced when these cells were seeded at low $(1 \times 105$ and $1 \times 104)$ cell density.

hMSCs seeded in constructs expressed ischemic markers in vivo

The prevalence of dead hMSCs in the core of the constructs of interest of the present study strongly suggested that ischemia is the main cause responsible for the observed loss of implanted cells. To validate that the implanted cells were exposed to ischemia, expressions of three ischemia-induced markers, specifically, HIF-1 $\alpha$ (hypoxia), LDH-A (anaerobic metabolism), and BNIP3 (autophagy), were monitored on explants sections from the constructs seeded with $1 \times 106$ cells. At day 0 , hMSCs seeded in the constructs expressed these three markers but at different levels of intensity. At that time, HIF$1 \alpha$ expression was mainly cytoplasmic but nuclear staining was observed in $<10 \%$ of these cells (Fig. 4A, B). In contrast, all cells within the constructs exhibited strong LDH-A (Fig. 4C, D) and BNIP3 (Fig. $4 \mathrm{E}, \mathrm{F})$ protein expression at the cytoplasmic level. After implantation, the three ischemic markers tested were still expressed up to day 6 postimplantation: $70 \%-85 \%$ of the cells in the construct displayed an HIF- $1 \alpha$ cytoplasmic staining while nuclear staining appeared simultaneously with an accentuated cytoplasmic expression (Fig. 4A, B). Similarly, $74 \%-90 \%$ of the cells expressed LDH-A (Fig. 4C, D). Expression of the autophagic marker decreased with cell location since $26 \%$ of the cells within the outer area and $56 \%$ of the cells in the core of the construct exhibited cytoplasmic BNIP3 labeling $(p<0.05)$. Expression of the three ischemic markers tested strongly decreased after 15 days (Fig. 4B, D, F) and disappeared at 30 days postimplantation (data not shown). Immunostaining of tissue sections from unseeded constructs explanted 6 and 15 days postimplantation was also performed to assess the expression of ischemic markers by the invading host tissue (Supplementary Fig. S5). The absence of specific cell labeling for the HIF-1 $\alpha$, LDHA, or BNIP3 proteins provided evidence that the host cells did not express the ischemic markers; expression of the observed ischemic markers was, therefore, related to the implanted cells. 


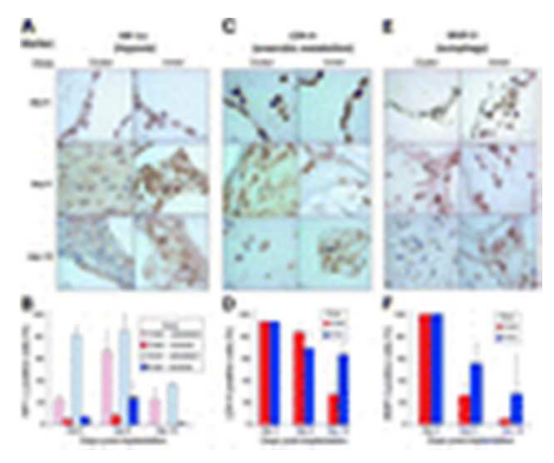

FIG. 4. The hMSCs within the constructs tested expressed ischemic markers. Sections of constructs seeded with $1 \times 106$ cells, either nonimplanted (day 0 ) or implanted for 6 and 15 days, were immunostained for (A) hypoxia-inducible factor-1 alpha (HIF-1 $\alpha$ ), (C) lactate dehydrogenase-A (LDHA), and (E) Bcl-2/adenovirus E1B $19 \mathrm{kDa}$-interacting protein 3 (BNIP-3) proteins. Stained cells were visualized in either the outer ( $<500 \mu \mathrm{m}$ of depth) or in the inner $(>1000 \mu \mathrm{m}$ of depth) areas. The number of (B) HIF-1 $\alpha$, (D) LDH-A, and (F) BNIP-3-positive cells per field present in either the outer (red bars) or inner area (blue bars) of the constructs seeded with $1 \times 106$ cells were counted. HIF-1 $\alpha$ positive cells stained at the cytoplasmic level were distinguished from those cells stained at both the cytoplasmic (pink and light blue) and nuclear levels (dark red and dark blue). For each marker examined, the stained cells were counted in five fields from each area of each section (corresponding to $>100$ observed cells). The data were expressed as mean \pm SEM. Color images available online at www.liebertpub.com/tea

Comparison of stainings for each ischemic marker by cells present in the inner and outer parts of the constructs evidenced that the intensity was stronger, and the frequency was higher, in areas located deeper in the construct. The numbers of HIF- $1 \alpha$-labeled cells at both the cytoplasmic and nuclear levels in the inner construct area were higher than those present in the outer area at all time points tested (Fig. 4B). At day 6 and 15, staining of LDH-A and BNIP3, respectively, attenuated in cells located in the outer area but remained high in cells located in areas still containing remnant fibrin gel in the core of the construct (Fig. 4C, F).

These results provided evidence that hMSCs seeded in the constructs tested expressed the hallmarks of ischemia before and after their implantation. In addition, these data also provided evidence that hMSCs experienced two distinct levels of ischemia depending on where they were located in the outer or in the inner part of the construct; the ischemic stress in the core of the constructs was the strongest.

hMSCs in the constructs tested remained viable up to 15 days postimplantation

Histological analysis of specimens stained with HES (Supplementary Fig. S6) provided evidence for the development of vascular networks originating at the host tissue that colonized the periphery of the implant ( $<500 \mu \mathrm{m}$ of depth) as early as 6 days postimplantation. The vascularization process continued over time, entirely invaded the outer part of the implanted construct after 15 days and the whole construct after 30 days. At day 15 postimplantation, the outer area ( $<500 \mu \mathrm{m}$ of depth) displayed the following features: (i) the number of TUNEL-positive cells present in this area was insignificant (Fig. 3I); (ii) the number of GFP-positive cells was still high (Fig. 3H); and (iii) this area was reperfused (invaded by neovascular networks). However, these remaining hMSCs irremediably continued to disappear at later (up to 30 days) time points evidenced by the loss of the respective BLI signal during those times. This result raises the question whether the hMSCs remaining at day 15 
were still viable or experienced a primary fatal metabolic stress that would lead to their subsequent death. To elucidate this aspect, the proliferative potential of these cells was compared to that of hMSCs present in TE constructs at earlier (day 2 and day 6) time points and to hMSCs that were not seeded onto scaffolds. TE constructs were explanted after 2, 6, and 15 days postimplantation and cells from each construct were recovered and cultured under standard in vitro tissue culture conditions. The average numbers of hMSCs recovered from each explant (estimated by the BLI signal emitted 1 day postseeding and compared to a standard curve of luciferase labeled-hMSC) were 53,000, 123,000, and 10,000 Luc-GFP-MSCs for the TE constructs explanted at day 2, 6, and 15 postimplantation, respectively. The proliferation rates of explanted cells were comparable in all groups tested and exhibited a delay of the exponential phase for the group explanted at day 15 (Fig. $5)$. This delay was explained by the low Luc-GFP hMSC seeding density $(<10,000$ cells/cm2) resulting from their low number recovered from day 15 TE constructs; indeed, a study of cell proliferation starting with different seeding densities demonstrated that nonimplanted hMSCs seeded at $<10,000$ cells/cm2 did not proliferate over an 18-day period of culture (Fig. 5 insert). These results provided evidence that hMSCs present in TE constructs for up to 15 days postimplantation were viable and displayed a similar proliferative potential as the nonimplanted hMSCs cultured under standard ( $21 \%$ O2) cell culture conditions.

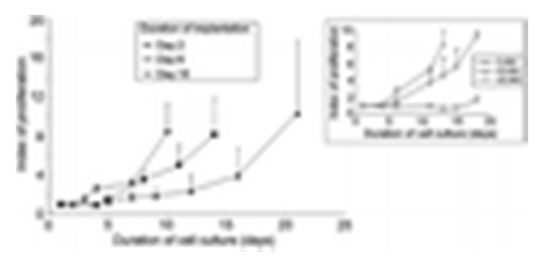

FIG. 5. The hMSCs present in the constructs tested remained viable for up to 15 days postimplantation. In vitro cell proliferation of Luc-GFP-hMSC extracted from constructs explanted 2, 6 , and 15 days postimplantation was determined. Cell proliferation was monitored noninvasively using BLI after addition of luciferin substrate to the cell cultures. The "index of proliferation" was defined as the photon flux from the cells present in each well measured at each time point normalized to that measured at day 1 postseeding. Insert: Time course of in vitro cell proliferation of Luc-GFP-hMSC seeded at 5000, 10,000, and 20,000 cells/cm2. The data were expressed as mean $\pm S E M ; n=8$ constructs for each duration of implantation tested and $n=3$ for each cell seeding density tested.

\section{Discussion}

Massive cell death observed in tissue constructs in vivo is a major impediment to their ultimate biological functionality. Despite the crucial need to better understand the biological and biochemical cascades that lead to such massive cell loss, only few studies that assess the death of implanted progenitor cells in a tissue engineering perspective have been reported in the literature. Local tissue ischemia is considered a prime cause for such accelerated cell mortality but it has never been clearly demonstrated in TE constructs so far. The present study was an attempt to fill this gap of knowledge. Ischemia originates from inadequate vascularization within implanted constructs; absence of preexisting vascular networks provokes an environment of low oxygen tension and nutrients and growth factors deprivation that implanted cells encounter in constructs; such metabolic stress strongly affects cell survival. To assess the impact of ischemia on the loss of implanted hMSCs as a function of their location in implanted constructs, we hereby investigated the in vivo temporal and spatial fate of 
hMSCs within a fibrin gel/coral ceramic scaffold in an ectopic mice model. The present study focused on the early ( $<30$ days) fate of implanted cells. For this reason, assessment of the osteogenic ability of the tested tissue constructs was outside the scope of this study. Fibrin gel was selected and used as the scaffold material because it promotes hMSC adhesion, spreading, and angiogenesis and wound healing processes.26,27 Coral ceramic also promotes cell adhesion, proliferation and osteogenic differentiation of bone marrow-derived stem cells.7,28 In the present study, the hMSC SV56 cell adhesion and proliferation on this scaffold was checked and confirmed in vitro. Based on these results, any causal effect of cell loss due to the composite scaffold biomaterial used was excluded. However, because some aspects of the material substrate, such as physicochemical features, size, shape, and porosity may critically affect cell functions pertinent to new tissue formation (including survival of grafted cells), the results of the present study cannot be directly compared to those from studies using another scaffold material.

Foremost, our results confirmed massive death of hMSCs distributed in coral particles/fibrin gel constructs over time after subcutaneous implantation in nude mice. The number of viable implanted cells decreased consistently over the 30 -day period of the study; as a result $<1 \%$ of the initial viable cell number remained at that time. Our results are in agreement with those by other researchers who reported overall loss when MSCs were contained in ceramic scaffolds and implanted either ectopically or orthotopically in rodents.8-10,12,13,29 The reported rate of transplanted cell loss varied (from 70 to 100\%) within the first month after implantation. Such variations may be explained by differences in MSC origin (species and tissue origin), the number of transplanted cells, the scaffold material used, the anatomical site of implantation, and the immune tolerance of the implantation model (syngenic vs. xenogenic).8,10,12,13,29,30 In the present study, we investigated the fate of an immortalized hMSC cell line after ectopic implantation in a xenogenic model. Using the same construct model, we recently examined the fate of primary Luc-GFP mMSC (harvested from Luc-GFP transgenic mice) after implantation in a syngenic model and observed similar massive (>90\%) disappearance of the implanted mMSC over the 3-week period of implantation (unpublished data). Similar results were reported by Tasso et al.,10 and more recently by Boukhechba et al.,30 who showed that grafted bone marrow-derived mouse MSCs did not survive $>3$ weeks after implantation in a syngenic model of ectopic bone regeneration. At this time, however, it is not known whether the short-term cell survival of bone marrow-derived MSC observed in mice may occur in the clinical milieu in autologous cell-based tissue engineering applications. Direct extrapolation to clinical use/applications needs to take into account the specific properties of human bone marrow-derived MSCs after autologous implantation.

With the exception of the study by Giannoni et al.9 who reported a peak of apoptosis (evidenced by TUNEL staining of explant sections) of sheep MSCs (distributed in ceramic/fibrin gel scaffolds) at day 2 postimplantation in a similar nude mouse model, however, none of the other studies clearly proved the death of implanted cells; the studies reported either extinction of the BLI signal from Luc-labeled cells contained in constructs or the disappearance of GFP-labeled cells in constructs but not definitive proof of cell death. In contrast, the present study provided evidence of massive cell death by TUNEL labeling of explanted tissue sections and determined that cell death peaked at 15 days and remained elevated at 30 days postimplantation.

Additionally, the effect of the number of seeded cells on their survival upon implantation was assessed: for all cell densities tested, the long-term viability of hMSCs on the TE constructs irremediably declined over time for up to 30 days postimplantation. The present study provided evidence that there is an optimal cell seeding density (specifically, $1 \times 106$ cells per construct tested) that resulted in the most "favorable" cell survival during the first week postimplantation. In contrast, 
either too low $(1 \times 104)$ or too high $(5 \times 106)$ cell seeding densities in the constructs tested accelerated cell death. Although few, some literature reports provided evidence that seeding MSCs at high densities ( $>1 \times 106$ cells) in constructs improved the in vivo viability of these cells.13,29 Several studies reported that grafted MSCs act as trophic factors and secrete several bioactive pro-survival paracrine factors.31-33 In the present study, the constructs that contained low $(1 \times 104)$ cell numbers, displayed sparse GFP cells distributed throughout the implanted constructs suggesting that the amount of secreted paracrine factors by the implanted cells was weak to promote their survival in vivo. On the other hand, the fact that cells seeded at high $(5 \times 106)$ density died at the same rate as those seeded at low $(1 \times 104)$ density, suggests that large numbers of cells within scaffolds may consume fast available nutrients (glucose and amino acids) and oxygen and also contribute to local accumulation of cell metabolic waste products. 34 The results of the present study indicated that an optimal cell density must be defined for each type of TE construct to maintain cell viability in vivo.

Expression of the hypoxia-regulated HIF1 $\alpha$ transcription factor and two of its downstream regulated proteins, specifically, LDH-A and BNIP3, provided evidence that the implanted cells of the present study were exposed to ischemia. Cells seeded in the constructs tested (and cultured for $24 \mathrm{~h}$ under standard tissue culture conditions) experienced hypoxia/ischemia before being implanted in animals (day 0 of the study). Upon implantation, the three ischemic markers tested were highly expressed up to day 6 . The high LDH-A levels of cells located in the constructs tested in the present study confirmed the switch-on of anaerobic metabolism resulting from the reduced oxygen environment. Further, the BNIP3 expression results proved induction of a pro-survival autophagic response.19 The three ischemic markers decreased at day 15 and were absent at day 30 postimplantation; in fact, they attenuated with kinetics commensurate with those of the invading host tissue.

We further assessed the fate of grafted cells as a function of their location within the constructs tested and found striking differences between the inner and the outer parts of the construct. In the core ( $>1000 \mu \mathrm{m}$ into the construct depth), cells died faster and predominantly ( $>50 \%$ of TUNELpositive cells were located in the inner area of the constructs); this result was enhanced when the cells were seeded at the low densities tested. Similarly, the frequency of positive cells and the intensity of staining for each ischemic marker tested significantly increased in areas deeper into the construct. All these results provided evidence that the primary metabolic stress encountered by hMSCs intensified in the core of the constructs tested; this evidence supports the explanation that ischemia (a harsh condition specifically found in areas located deep in the TE constructs) is responsible for the observed massive death of implanted cells in these inner construct areas.

In contrast, in the outer part of the constructs ( $<500 \mu \mathrm{m}$ of depth), few apoptotic cells were present at all postimplantation time points tested, while GFP-positive hMSCs were still present in high numbers up to day 15 postimplantation. At this time point, the outer construct area was invaded by vascular networks originating from the colonizing host tissue, while cell ischemic markers had either strongly weakened or disappeared, indicating that these outer areas were possibly reperfused. Nevertheless, monitoring cell viability (as determined by BLI and GFP-positive cell quantification) in the constructs tested provided evidence that the implanted cells irremediably continued to disappear at later time points (up to 30 days) postimplantation resulting in the entire loss of implanted cells from the inner and outer construct areas. To rule out the possibility that the implanted cells experienced a primary fatal metabolic stress that led to their death, hMSCs from TE construct explants were recovered and cultured under standard tissue culture conditions in vitro. Similar proliferative rates of hMSCs explanted at either day 15 or earlier and of nonimplanted hMSCs provided evidence regarding the viability of hMSCs in TE constructs up to day 15 postimplantation. Taken together, these observations support the explanation that the disappearance of hMSCs 
located in the outer area of implanted TE constructs at later time was due to a biochemical process distinct from ischemia.

Several possible explanations could be raised for the observed loss of implanted hMSCs after reperfusion. Among them, establishment of blood supply following ischemia reintroduced reactive oxygen species that may cause oxidative cell damage leading to loss of function and/or cell death.35 In addition, the invading vascular networks allow the exchange of molecules and cells of different type between the host and the implant, including immune-reactive effector cells. Although undifferentiated MSC populations have the potential to downregulate the immune response,36,37 one cannot exclude the possibility that the long-term disappearance of a part of implanted exogenous MSCs originated from activation of the host immune system. In the present study, histological examination of explants indicated the presence of some immune cells (including macrophages, polynuclear, and lymphocytes to a lesser extend) located in the host tissue invading the constructs at day 15 and day 30 postimplantation; this situation evoked the occurrence of a weak/moderate inflammatory and immune response against the cell constructs (data not shown). The newly formed vascular networks could also be the way by which implanted MSCs could leave the scaffold and reach other organs through the SDF-1/CXCR4 axis involved in the homing of MSC to injured tissues.38,39 In a similar ectopic model as the one used in the present study, Tasso et al. reported migration of exogenous murine MSC from the construct to the spleen.10 In the present study, the BLI signal (displayed in pseudocolor on BLI images taken over the entire period of implantation) remained strictly localized at the site of the implanted cell construct. Also, intraperitoneal injections of Luciferin substrate were performed in each tested animal at the last (30 days) postimplantation time point to check the potential migration of luc-labeled hMSC from the implanted site; the BLI images, however, did not provided evidence of BLI signal from any body part of the mice (data not shown). These observations excluded the possibility of a massive migration of implanted hMSCs out of the constructs but not the possibility of sparse migration of hMSCs that was not detectable by the $\mathrm{BLI}$ assay. Further research is needed to provide definitive answers to these issues. Nevertheless, understanding the cause of the death/disappearance of cells in the outer zone of implanted constructs is relevant in the bone TE field; in this case, ectopic new bone in implanted constructs was centripetally formed from the periphery toward the central areas of these constructs30,40,41; this observation highlights the potential role of MSCs located in these peripheral areas for new tissue growth.

Although ischemia appeared to be the prime cause, the results of the present study provided evidence that death/disappearance of implanted hMSC is the result of combined complex biological processes (still not fully understood) that need to be thoroughly elucidated. Strategies to enhance cell survival in vivo need to take into account these different, and perhaps additive, events.

\section{Conclusion}

The results of the present study provided evidence that, although the seeding density of hMSCs affected cell survival at early times ( $<15$ days), long-term ( 30 days postimplantation) viability of the TE constructs tested in vivo irremediably declined over time for all cell densities tested. Ischemia was the prime cause of the massive death of transplanted cells. Soon after cell construct preparation, the seeded hMSCs were under ischemia that increased after construct implantation in vivo and intensified in the core of the constructs where cells in great numbers died. Another biological mechanism (that still needs to be elucidated) seemed, however, to be responsible for the death/disappearance of viable implanted hMSCs remaining in the outer part of TE constructs once neovascular networks were established. Complete identification of conditions responsible for the 
death of implanted hMSCs in tissue-engineered constructs is needed to develop relevant strategies for improving hMSC survival in vivo.

\section{Acknowledgments}

The authors gratefully acknowledge Inoteb Inc. (Levallois-Perret, France) for donating the coral scaffolds and Baxter, Inc., for donating the Tissucol used in the present study. We would also like to thank Dr. R. Vallefuoco for his help with the histological analysis and Professor R. Bizios for critically reading the article. This project was supported by the French National Research Agency (ANR) through the TecSan program (project GLASSBONE $n^{\circ} 08-T E C S-004$ ), by the Centre National de la Recherche Scientifique (CNRS), and by the National French Academy of Medicine (ACB).

\section{Disclosure Statement}

No competing financial interests exist.

\section{References}

1. Charbord, P. Bone marrow mesenchymal stem cells: historical overview and concepts. Hum Gene Ther 21, 1045, 2010.

2. Cancedda, R., Mastrogiacomo, M., Bianchi, G., Derubeis, A., Muraglia, A., and Quarto, R. Bone marrow stromal cells and their use in regenerating bone. Novartis Found Symp 249, 133, 2003.

3. Logeart-Avramoglou, D., Anagnostou, F., Bizios, R., and Petite, H. Engineering bone: challenges and obstacles. J Cell Mol Med 9, 72, 2005.

4. Chung, C., and Burdick, J.A. Engineering cartilage tissue. Adv Drug Deliv Rev 60, 243, 2008.

5. Leclerc, T., Thepenier, C., Jault, P., Bey, E., Peltzer, J., Trouillas, M., Duhamel, P., Bargues, L., Prat, M., Bonderriter M, et al. Cell therapy of burns. Cell Prolif 44 Suppl 1, 48, 2011.

6. Sensebe, L., Krampera, M., Schrezenmeier, H., Bourin, P., and Giordano, R. Mesenchymal stem cells for clinical application. Vox Sang 98, 93, 2010.

7. Viateau, V., Guillemin, G., Bousson, V., Oudina, K., Hannouche, D., Sedel, L., Logeart-Avramoglou, D., and Petite, $\mathrm{H}$. Long-bone critical-size defects treated with tissue-engineered grafts: a study on sheep. J Orthop Res 25, 741, 2007.

8. Dennis, J.E., Haynesworth, S.E., Young, R.G., and Caplan, A.I. Osteogenesis in marrow-derived mesenchymal cell porous ceramic composites transplanted subcutaneously: effect of fibronectin and laminin on cell retention and rate of osteogenic expression. Cell Transplant 1, 23, 1992.

9. Giannoni, P., Scaglione, S., Daga, A., Ilengo, C., Cilli, M., and Quarto, R. Short-time survival and engraftment of bone marrow stromal cells in an ectopic model of bone regeneration. Tissue Eng Part A 16, 489, 2009.

10. Tasso, R., Augello, A., Boccardo, S., Salvi, S., Carida, M., Postiglione, F., Fais, F., Truini, M., Cancedda, R., and Pennesi, G. Recruitment of a host's osteoprogenitor cells using exogenous mesenchymal stem cells seeded on porous ceramic. Tissue Eng Part A 15, 2203, 2009. 
11. Jager, M., Degistirici, O., Knipper, A., Fischer, J., Sager, M., and Krauspe, R. Bone healing and migration of cord blood-derived stem cells into a critical size femoral defect after xenotransplantation. J Bone Miner Res 22, 1224, 2007.

12. Degano, I.R., Vilalta, M., Bago, J.R., Matthies, A.M., Hubbell, J.A., Dimitriou, H., Bianco, P., Rubio, N., and Blanco, J. Bioluminescence imaging of calvarial bone repair using bone marrow and adipose tissue-derived mesenchymal stem cells. Biomaterials 29, 427, 2008.

13. Geuze, R.E., Prins, H.J., Oner, F.C., van der Helm, Y.J., Schuijff, L.S., Martens, A.C., Kruyt, M.C., Alblas, J., and Dhert, W.J. Luciferase labeling for multipotent stromal cell tracking in spinal fusion versus ectopic bone tissue engineering in mice and rats. Tissue Eng Part A 16, 3343, 2010.

14. Liu, J., Barradas, A., Fernandes, H., Janssen, F., Papenburg, B., Stamatialis, D., Martens, A., van Blitterswijk, C., and de Boer, J. In vitro and in vivo bioluminescent imaging of hypoxia in tissueengineered grafts. Tissue Eng Part C Methods 16, 479, 2010.

15. Russ, A.L., Haberstroh, K.M., and Rundell, A.E. Experimental strategies to improve in vitro models of renal ischemia. Exp Mol Pathol 83, 143, 2007.

16. Loeuillet, C., Bernard, G., Remy-Martin, J., Saas, P., Herve, P., Douay, L., and Chalmers, D. Distinct hematopoietic support by two human stromal cell lines. Exp Hematol 29, 736, 2001.

17. Firth, J.D., Ebert, B.L., and Ratcliffe, P.J. Hypoxic regulation of lactate dehydrogenase A. Interaction between hypoxia-inducible factor 1 and cAMP response elements. J Biol Chem 270, 21021, 1995.

18. Levine, B. Eating oneself and uninvited guests: autophagy-related pathways in cellular defense. Cell 120, 159, 2005.

19. Bellot, G., Garcia-Medina, R., Gounon, P., Chiche, J., Roux, D., Pouyssegur, J., and Mazure, N.M. Hypoxia-induced autophagy is mediated through hypoxia-inducible factor induction of BNIP3 and BNIP3L via their BH3 domains. Mol Cell Biol 29, 2570, 2009.

20. Scarlatti, F., Granata, R., Meijer, A.J., and Codogno, P. Does autophagy have a license to kill mammalian cells? Cell Death Differ 16, 12, 2009.

21. Deroose, C.M., Reumers, V., Gijsbers, R., Bormans, G., Debyser, Z., Mortelmans, L., and Baekelandt, V. Noninvasive monitoring of long-term lentiviral vector-mediated gene expression in rodent brain with bioluminescence imaging. Mol Ther 14, 423, 2006.

22. Ibrahimi, A., Vande Velde, G., Reumers, V., Toelen, J., Thiry, I., Vandeputte, C., Vets, S., Deroose, C., Bormans, G., Baekelandt $\mathrm{V}$, et al. Highly efficient multicistronic lentiviral vectors with peptide $2 \mathrm{~A}$ sequences. Hum Gene Ther 20, 845, 2009.

23. Logeart-Avramoglou, D., Oudina, K., Bourguignon, M., Delpierre, L., Nicola, M.A., Bensidhoum, M., Arnaud, E., and Petite, $\mathrm{H}$. In vitro and in vivo bioluminescent quantification of viable stem cells in engineered constructs. Tissue Eng Part C Methods 16, 447, 2010.

24. Guillemin, G., Meunier, A., Dallant, P., Christel, P., Pouliquen, J.C., and Sedel, L. Comparison of coral resorption and bone apposition with two natural corals of different porosities. J Biomed Mater Res 23, 765, 1989.

25. Guillemin, G., Patat, J.L., Fournie, J., and Chetail, M. The use of coral as a bone graft substitute. J Biomed Mater Res 21, 557, 1987. 
26. Bensaid, W., Triffitt, J.T., Blanchat, C., Oudina, K., Sedel, L., and Petite, H. A biodegradable fibrin scaffold for mesenchymal stem cell transplantation. Biomaterials 24, 2497, 2003.

27. Janmey, P.A., Winer, J.P., and Weisel, J.W. Fibrin gels and their clinical and bioengineering applications. J R Soc Interface 6, 1, 2009.

28. Petite, H., Viateau, V., Bensaid, W., Meunier, A., de Pollak, C., Bourguignon, M., Oudina, K., Sedel, L., and Guillemin, G. Tissue-engineered bone regeneration. Nat Biotechnol 18, 959, 2000.

29. Olivo, C., Alblas, J., Verweij, V., Van Zonneveld, A.J., Dhert, W.J., and Martens, A.C. In vivo bioluminescence imaging study to monitor ectopic bone formation by luciferase gene marked mesenchymal stem cells. J Orthop Res 26, 901, 2008.

30. Boukhechba, F., Balaguer, T., Bouvet-Gerbettaz, S., Michiels, J.F., Bouler, J.M., Carle, G.F., Scimeca, J.C., and Rochet, N. Fate of bone marrow stromal cells in a syngenic model of bone formation. Tissue Eng Part A 17, 2267, 2011.

31. Kinnaird, T., Stabile, E., Burnett, M.S., Shou, M., Lee, C.W., Barr, S., Fuchs, S., and Epstein, S.E. Local delivery of marrow-derived stromal cells augments collateral perfusion through paracrine mechanisms. Circulation 109, 1543, 2004.

32. Kinnaird, T., Stabile, E., Burnett, M.S., Lee, C.W., Barr, S., Fuchs, S., Epstein, S.E. Marrow-derived stromal cells express genes encoding a broad spectrum of arteriogenic cytokines and promote in vitro and in vivo arteriogenesis through paracrine mechanisms. Circ Res 94, 678, 2004.

33. Chen, L., Tredget, E.E., Wu, P.Y., Wu, Y. Paracrine factors of mesenchymal stem cells recruit macrophages and endothelial lineage cells and enhance wound healing. PLoS One 3, e1886, 2008.

34. Muschler, G.F., Nakamoto, C., Griffith, L.G. Engineering principles of clinical cell-based tissue engineering. J Bone Joint Surg Am 86-A, 1541, 2004.

35. Ryter, S.W., Kim, H.P., Hoetzel, A., Park, J.W., Nakahira, K., Wang, X., Choi, A.M. Mechanisms of cell death in oxidative stress. Antioxid Redox Signal 9, 49, 2007.

36. Le Blanc, K., Ringden, O. Mesenchymal stem cells: properties and role in clinical bone marrow transplantation. Curr Opin Immunol 18, 586, 2006.

37. Uccelli, A., Moretta, L., Pistoia, V. Mesenchymal stem cells in health and disease. Nat Rev Immunol 8, 726, 2008.

38. Bensidhoum, M., Chapel, A., Francois, S., Demarquay, C., Mazurier, C., Fouillard, L., Bouchet, S., Bertho, J.M., Gourmelon, P., Aigueperse J and others. Homing of in vitro expanded Stro-1- or Stro-1+ human mesenchymal stem cells into the NOD/SCID mouse and their role in supporting human CD34 cell engraftment. Blood 103, 3313, 2004.

39. Fong, E.L., Chan, C.K., Goodman, S.B. Stem cell homing in musculoskeletal injury. Biomaterials 32, 395, 2011.

40. Cooper, L.F., Harris, C.T., Bruder, S.P., Kowalski, R., Kadiyala, S. Incipient analysis of mesenchymal stem-cell-derived osteogenesis. J Dent Res 80, 314, 2001.

41. Kruyt, M.C., Dhert, W.J., Oner, F.C., van Blitterswijk, C.A., Verbout, A.J., de Bruijn, J.D. Analysis of ectopic and orthotopic bone formation in cell-based tissue-engineered constructs in goats.

Biomaterials 28, 1798, 2007. 